\title{
Carnets
}

Revue électronique d'études françaises de l'APEF

Deuxième série - $16 \mid 2019$

Le Récit inachevé: études sur Mai 68

\section{Faut-il 'achever' mai 68 ? Enjeux et réflexions}

João Domingues

\section{(2) OpenEdition}

\section{Journals}

\section{Édition électronique}

URL : http://journals.openedition.org/carnets/9694

DOI : 10.4000/carnets.9694

ISSN : 1646-7698

Éditeur

APEF

\section{Référence électronique}

João Domingues, «Faut-il 'achever' mai 68 ? Enjeux et réflexions », Carnets [En ligne], Deuxième série 16 | 2019, mis en ligne le 31 mai 2019, consulté le 09 novembre 2019. URL : http:// journals.openedition.org/carnets/9694; DOI : 10.4000/carnets.9694

Ce document a été généré automatiquement le 9 novembre 2019.

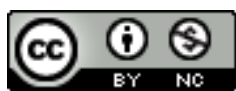

Carnets est mis à disposition selon les termes de la licence Creative Commons - Atribution - Pas d'utilisation commerciale 4.0 International. 


\title{
Faut-il 'achever' mai 68 ? Enjeux et réflexions
}

\author{
João Domingues
}

1 Avant de me demander "s'il faut achever mai 68 " et d'entamer une réflexion sur le sujet, je me suis interrogé sur la pertinence d'une telle question à un moment où, depuis dix ans au moins, de grands intellectuels débattent de ce qui nous reste de Mai $68^{1}$. Au cours d'un long débat à la Bibliothèque nationale de France, en mars 2008, Luc Ferry et Edgar Morin ont analysé ce mouvement étudiant devenu, comme on le sait, une crise sociale et politique lourde de conséquences, non seulement dans ses changements politiques ${ }^{2}$, mais surtout dans les changements de paradigmes moraux et culturels qu'il a insufflés, notamment le rapport à l'autorité et les rapports civiques, la liberté d'expression, les droits de la femme et l'ouverture au monde. Or, pour le meilleur et pour le pire, force est de constater que la plupart de ces changements travaillent encore notre société. Car, s'il est vrai que bien des idées, sorties de ce long mouvement, perdurent, il n'en demeure moins légitime de chercher, parmi ses conséquences, certains excès, certaines contraintes, certains bornes, voire de sonder les ombres de ce mouvement, resté à bien des égards imparfait ; et si la formule « métro boulot dodo » ne rendait pas compte d'une vie parfaite - loin s'en faut -, «demander l'impossible» ne semble pas, non plus, le dessein le plus raisonnable à poursuivre quand on se propose de construire un monde plus juste, plus équitable, plus libre ou simplement meilleur. À moins qu'on l'entende à la manière des surréalistes pour qui « demander l'impossible » pouvait signifier demander à l'imagination de nous rendre réel l'impossible, justement.

2 Lors d'un meeting pendant la campagne électorale de 2007, le slogan «Vivre sans contraintes, jouir sans entraves » a mérité de la part de Nicolas Sarkozy l'inférence que nous connaissons tous :

Puisqu'il n'y a plus de règles, plus de normes, plus de morale, plus de respect, plus d'autorité, puisque tout se vaut, alors tout est permis [...]. Les héritiers de mai 68 ont abaissé le niveau moral de la politique. ${ }^{3}$

3 Et avait-il ajouté : «Voilà pourquoi il fallait tourner cette page » ... Comme si Mai 68 était responsable de tous les maux de la France actuelle et notamment du capitalisme 
libéral le plus effréné, dans lequel il n’y a, justement, plus de règles, plus de normes, plus de morale, plus de respect pour l'être humain.

4 "Achevons mai 68 ", rétorque Michel Onfray ${ }^{4}$, mais non à l'image d'une bête malade ou d'un vieux rêve... Michel Onfray reconnait que beaucoup des bâtisseurs de Mai 68 ont vite plongé dans «l'européanisme libéral», dans "l'insurrection internationale de salon, à Saint Germain des Prés, de l'inspection générale, des grands corps d'Etat... ». D'autres, toujours selon Onfray, sont devenus socialistes, reconvertis du trotskisme ou du maoïsme, mais faisant «de la classe ouvrière le cadet de leurs soucis ». D'autres encore sont devenus plus américanophiles qu'ils n'avaient été soviétophiles et défenseurs acharnés de Lénine. On les retrouve aujourd'hui bien installés dans la société civile française. Or, pour reprendre Michel Onfray, aucun ne l'a achevé ; ils ont, de fait, tous plus ou moins renoncé à Mai 68, et leur histoire est l'histoire de cette contre révolution qu'est le capitalisme débridé, c'est-à-dire la trahison même de Mai 68.

5 Pour Michel Onfray, s'il faut 'achever' Mai 68, c'est « en ajoutant au travail du négatif que fut ce Mai interrompu la positivité de valeurs nouvelles - telles que l'altermondialisme, le féminisme, l'écologie, ... les économies solidaires... ». Ce serait alors achever ce mouvement au lieu d'en finir avec, de ne plus en parler, comme si cela ne voulait plus rien dire, comme s'il n'y avait plus rien à dire, comme s'il fallait vraiment « tourner cette page ».

6 Or, qu'on le veuille ou pas, et indépendamment de l'idéologie sous-jacente à cet observateur de notre société, cette page reste, nous semble-t-il, grande-ouverte. On est bien loin d'avoir achevé Mai 68 : la sensibilité sociale et la pensée engendrées par ce mouvement, social et idéologique, a encore bien des choses à dire ; l'histoire a un rôle à jouer pour démystifier, mieux éclairer et témoigner de ce que fut vraiment ce mouvement unique d'union de presque toutes les classes quelles que soient leurs origines et leurs provenances sociales, dans une impulsion de mécontentement généralisé - et ce malgré le succès des "trente glorieuses » -, et de contestation du statu quo. Quoi qu'il en soit, Mai 68 restera dans l'histoire comme une période qui a profondément marqué la vie et la pensée françaises, et il mérite qu'on s'y attache et qu'on le repense.

7 Ne voulant pas faire l'éloge gratuit tout en évitant la nécrologie, nous suivons ici les réflexions d'un autre critique non moins problématique de Mai 68, Alain Finkielkraut. Nous partons des dérives et des incomplétudes de mai 68 qu'il pointe dans ses critiques pour mieux identifier ce qu'il reste à dire ou à faire pour que Mai 68 continue d'interroger notre pensée et notre action, en France, en Europe et ailleurs. Tel est, nous semble-t-il, le but essentiel de la célébration du cinquantenaire d'un mouvement qui, comme nous le savons, a, en quelque sorte, libéré la société française du poids de certains tabous, qui a tant fait pour les droits de la femme, qui a voulu réorienter, tant bien que mal, la jeunesse et qui porte en lui, qu'on le veuille ou pas, une compréhension propre de la destinée humaine, de l'homme et du monde.

\section{I. « II est interdit d'interdire »}

8 Slogan majeur des événements de Mai 68 et symbole de la rébellion des étudiants contre le conservatisme de l'époque, ce cri de ralliement est peut-être celui qui fait aujourd'hui le plus débat et qu'il faudrait peut-être repenser. Loin de moi l'idée de 
laisser entendre que, en exhibant ce slogan, la génération de 68 est la seule responsable de l'individualisme exacerbé et de la violence dont on souffre aujourd'hui. Pourtant, face à la violence gratuite, aux agressions entre étudiants et contre les enseignants dans certaines écoles, on ne peut que constater un retour aux valeurs d'autorité et de fermeté, parfois au détriment de cette liberté tant désirée, de cette envie d'interdire tout interdit.

Ce n'est pas interdit, mais il est peut-être lamentable que «l'animatrice chargée de la météo sur une chaîne de télévision renommée [...] annonce qu'il pleuvra en plein mois de mai sur le festival de Cannes par ces mots: 'un temps de merde' (Finkielkraut, 2013 : 151). En 2012, le journal Libération ne s'est pas interdit non plus d'enregistrer la «maxime de l'escrimeur français Gauthier Grumier qui "se lâcha» en disant: "En chier, jusqu'à la perfection» (Finkielkraut, 2013: 151). Ce n'est pas interdit, et le problème, comme l'a fort bien observé Finkielkraut, n'est pas la vulgarité mais l'usage de ces vulgarités en public sans que cette vulgarité ne soit même plus perceptible. Personne ne réagit ; personne ne s'en inquiète, à aucun niveau de la société, en aucune circonstance. Et il cite un autre exemple flagrant: « Daniel Cohn-Bendit a eu en public ce compliment envers Ségolène Royal: c'« est une vraie soixante-huitarde. Elle dit: “quand ça me fait chier, je m'en vais" (Finkielkraut, 2013: 153). Que le soixantehuitard le dise en privé peut être compréhensible, à la limite acceptable, mais que l'homme politique, donc public, puisse s'exprimer ainsi traduit un manque de respect certain envers son public et envers lui-même, sans vouloir l'accuser de manque d'éducation civique. Cohn-Bendit est sensé posséder d'autres moyens d'expression pour dire ce qu'il pense.

10 Car la société française reste soucieuse des libertés prises avec le langage. En effet, lorsqu'en novembre 2005 N. Sarkozy a dénoncé publiquement la «racaille» - qu'il faudrait, selon lui, "passer au karcher »-, on a aussitôt sursauté dans la presse pour désapprouver ce langage et rappeler que les dirigeants politiques n'étaient pas exemptés d'éducation civique (cf.: Le Monde, novembre 2005)5. La France n'est donc pas sourde ni indifférente; les Français cultivent encore certaines valeurs, apprécient une certaine pudeur, et gardent un sens du respect qu'ils tiennent à conserver aussi dans le langage.

11 On ne peut s'empêcher de dire que, sur ce point, Finkielkraut voit juste. Et il voit juste aussi lorsque, à propos de la liberté d'expression, il commente :

[...] Tout comme la censure, sa grande ennemie, cette liberté a sa part d'ombre. [...] 'Un homme, ça s'empêche', dit Camus, et s'il ne s'empêche pas tout seul, il faut des lois pour le contraindre et le punir. Pas de démocratie sans liberté. Pas de civilisation sans une pratique éclairée de la censure, comme le démontre a contrario l'anarchie barbare qui sévit sur la Toile. (Finkielkraut, 2015 : 262-263)

\section{II. « Jouir sans entraves »}

12 Avec les années 68 et dans les années suivantes, on a vu une certaine «morale hédoniste triompher: [...] nul n'entendait céder sur son désir, [...] les sexualités minoritaires sortaient de la clandestinité et accédaient fièrement à l'air libre " (Finkielkraut, 2015 : 15-16). La " jouissance sans entraves » n'était pas interdite, mais dans la pratique ce n'est pas si simple. Les libertés sexuelles, par exemple, tout en permettant l'épanouissement du corps humain - de l'homme et de la femme - et du 
plaisir que l'on peut s'en procurer, tournent bien souvent à la déception, parfois à des états pathologiques ou tout simplement à la maladie physique. Julia Kristeva raconte, à propos, à la première personne :

J'ai connu une étudiante [...], arrivée vierge et pudique à Paris, qui se lança à corps perdu dans le «sexe de groupe » des années 68 [...]. Or, quelques mois plus tard [...] je l'ai retrouvée à l'Assistance publique, atteinte d'une maladie des poumons (Kristeva, $1988: 48$ ).

13 Même si l'on peut rétorquer qu'un cas ne suffit pas à démontrer une règle, on ne peut s'empêcher d'observer que, dans ce domaine comme dans d'autres, cet hédonisme effréné, qui, tout compte fait, n'est plus qu'une envie de consommation sans bornes, participe souvent d'un repli sur soi, de l'égoïsme pur, borné et mesquin, et n'a plus rien à voir avec l'ouverture à l'autre, à l'épanouissement de soi, essentiel à la réalisation de son être. Par conséquent, il n'est pas étrange que ces mêmes jeunes gens recourent à des moyens assez individualistes pour se cultiver, et ne veuillent que s'amuser et vivre confortablement chez eux. Il n'est pas étrange non plus que ce 'confort bourgeois' soit devenu le but ultime de tant de jeunes résignés, qui avaient pourtant crié : «soyons réalistes, demandons l'impossible». Il ne s'agit donc pas d'un achèvement de mai 68 mais d'une dérive.

\section{III. "Soyons réalistes, demandons l'impossible"}

14 Ce slogan, attribuée à Guevara et repris par les étudiants contestataires français de Mai 68, est, plus qu'un rêve, le symbole de la frustration de toute une partie de la population qui passe de la résignation à la lutte indignée. Or, parmi ces indignés, et qui plus est compagnon de Guevara pour un temps, on comptait Régis Debray, devenu, comme on le sait, le premier des intellectuels critiques à vilipender les soixantehuitards et leur mythologie, parlant sans ambages d'une génération égoïste. Selon lui, Mai 68 est tout simplement le berceau de la nouvelle société bourgeoise.

Sans pour autant rien renier de sa jeunesse révolutionnaire, cet ancien compagnon de route de Guevara en vient à admettre que « l'homme a des racines, une généalogie, une mémoire ethnique, bref, qu'il ne se définit pas seulement par ses intérêts et par ses espérances » (Finkielkraut, 1987 : 115). Sans renoncer à l'idéal de fraternité, Debray reconnait pourtant comme une évidence que «l'homme a beau faire et beau dire pour liquider la morale de clan: ses maximes universalistes ne sont que de «luxueuses abstractions » (ibid. : 116) ${ }^{6}$.

\section{IV. «CRS = SS », on ne le dit plus, maintenant}

On ne le dit plus; au contraire, "il est inadmissible, affirme Primo Levi, de mettre un signe d'équivalence entre l'expérience de l'ouvrier qui travaille chez Fiat (disons, chez Renault en 1968), et le détenu d'Auschwitz, parce que le premier peut quitter son usine, le second ne peut sortir librement de son camp " (Camon, 1989: 19-20). Et Todorov d'ajouter sans détours : "Il est stupide de crier "CRS, SS". La différence est maximale dans l'expérience vécue et l'on n'a pas le droit de l'ignorer » (Todorov, $2017: 305$ ).

On ne le dit plus, en fait, et aucun soixante-huitard n'aurait aujourd'hui le courage de le répéter si ce n'est pour se rappeler le rythme ironiquement presque militaire de la 
contestation - C.R.S : SS ; CRS : SS. Au contraire, au moment des attentats contre le journal Charlie Hebdo, "le 11 janvier 2015, quatre millions et demi de Français ont acclamé les CRS et chanté la Marseillaise » (Finkielkraut, 2015 : 230). Le moment n'est pas le même, car l'histoire ne se répète pas, et les enjeux politiques et sociaux non plus ; mais le fait est que, cette fois, au lieu de contester le système dénonçant la répression, au lieu de célébrer la transgression, ou la subversion dans un esprit typiquement soixante-huitard, on en vient à applaudir les forces de l'ordre et à chanter la Marseillaise.

18 Une fois de plus la situation n'est pas simple, car «[...] il est difficile de ne pas remarquer que (lors de cet applaudissement des forces de l'ordre comme dans ce chant patriotique) les banlieues ne sont pas descendues dans la rue » (Finkielkraut, 2015 : 230), observe Finkielkraut presque sournoisement. Les Français sont descendus dans la rue et ils ont chanté la Marseillaise, peut-être parce que « la France découvre soudain, toutes sensibilités confondues, que sa manière d'être est attaquée et mise en péril » (Finkielkraut, 2015 : 231). En revanche, les jeunes des cités et les quartiers populaires, eux, ont manqué ce rendez-vous : les uns parce qu'ils ne se sont pas sentis concernés par l'esprit Charlie Hebdo, les autres parce que, pour eux, «les vengeurs du Prophète » sont « des héros, morts les armes à la main » (Finkielkraut, 2015 : 232). Voilà ce qui nous semble très grave, car cela signifie que d'autres formes d'oppression sont encore présentes et doivent être dénoncées. Nous pensons en effet que ces événements, loin d'incarner la dénégation de mai 68 , constituent au contraire la preuve que la société française, telle qu'elle est en train de se composer, a besoin de cet esprit de solidarité de base, d'égalité des chances, d'égalité des genres, de jouissance positive, de liberté et de respect de l'autre, que le mouvement de 68, malgré tous les efforts et tous les changements clamés, n'a pas réussi à implanter.

19 "Cours, camarade, le Vieux Monde est derrière toi !", dit le slogan. Et la France n'a cessé de courir depuis ; mais à présent, comme essoufflée, elle ralentit ... elle se regarde et ne reconnait plus son Vieux Monde. Aujourd'hui, à Paris, au lieu de jouir de l'égalité des genres ou de demander plus de liberté, certaines filles refusent encore de suivre les mêmes cours de gymnastique ou de piscine que les garçons ; certains enfants refusent de manger à la cantine pour des raisons religieuses, même si des plats de substitution sont prévus; et des conflits émergent au sein même de l'école ( $c f$. Finkielkraut, 213 : 19-20). Dans ce contexte, l'explication par les seules différences économiques et sociales qui rendraient l'intégration difficile ne tient donc pas compte de la discordance des usages et des différentes façons de concevoir la vie en société ${ }^{7}$, le "mieux vivreensemble » dans « la diversité et la mixité sociale » s'en trouvant de fait affaibli. Et la violence même, caractéristique de la France du début de ce XXIe siècle, ne découle pas tant de la révolte contre les inégalités ou de la soif d'acquisition que du désir d'être respecté et du sentiment de ne pas l'être (cf. Finkielkraut, $2013: 162$ ).

Depuis 1968, la " France a changé, la vie a changé, le changement lui-même a changé » (cf. Finkielkraut, $213: 22$ ), car, n'étant plus le produit de notre volonté, il est plutôt subi qu'entrepris ou désiré. Disons simplement, et pour abréger, que, cinquante ans plus tard, d'autres entraves ont surgi en France qui empêchent l'épanouissement d'une France multiculturelle et démocratique de l'être humain, et que, quoi qu'on en dise, Paris X, à Nanterre, est toujours à mille lieues de la Sorbonne. Il reste donc à (re)faire tout un 'Mai 68', peut-être en d'autres termes, mais il reviendra un jour, nécessairement. 


\section{L'avenir de Mai 68}

21 tant que mouvement idéologique consistant, l'avenir de mai 68 ou de son héritage aujourd'hui semble bien sombre. Un auteur comme Lipovetsky, dans son œuvre L'Ère du vide (1983) dans laquelle il développe l'idée du nihilisme des valeurs introduites par Mai 68, précise, par exemple, que ce mouvement n'avait pas de visée concrète sur l'avenir, même si on y rêvait d'un avenir « radicalement neuf» (L. Ferry et A. Renaut, $1988: 87$ ). De facto, Mai 68 a engendré au moins un moment de rupture et par rapport aux valeurs, aux canons et aux pratiques sociales et par rapport aux discours.

Ce même auteur définit encore 'l'esprit de mai' comme un " ensemble de significations, de finalités, de revendications, d'attitudes et d'actions typiques de ce moment et ayant donné au mouvement sa véritable originalité historique " (Lipovetsky, 1988: 91). De mouvement collectif d'abord, animé par un idéal de solidarité - avec les travailleurs en grève, avec les manifestants emprisonnés ou avec le peuple vietnamien -, de mouvement révolutionnaire allié au prolétariat et "voué à la mission de casser l'histoire en deux» (Lipovetsky, 1988: 93), ce mouvement a fini par révéler « une explosion d'aspirations et de revendications de type explicitement individualiste luttant pour la liberté sans limites et l'autonomie de l'individu, l'individu autosuffisant et existant d'abord pour lui-même (cf. Lipovetsky, 1988 : 93).

Ce mouvement étudiant a pris une ampleur telle, et semblait porter en lui tellement de créativité sociale, qu'Edgar Morin l'a qualifié de "prodigieuse Commune étudiante » (Morin, 1988: 13 ). Mais il n'en demeure pas moins vrai que, tout en s'insurgeant avec leur radicalisme antiautoritaire contre toutes les tutelles monopolisant la vie sociale qu'elles soient gaullistes, communistes ou autres -, nous sommes portés à croire que, dans ces circonstances, le sens de la société n'est plus centré sur le collectif, mais bien sur l'individu qui se doit d'inventer de nouvelles relations sociales. C'est peut-être là un des grands échecs de Mai 68, c'est-à-dire le fait qu'il n'a pas réussi à inverser la réalité dénoncée ni à créer un nouvel ordre social (cf. Aron, $1968: 14$ ).

\section{En conclusion}

Les événements de Mai 68, pendant lesquels étudiants et ouvriers ont convergé dans un même désir d'émancipation, ne peuvent être confinés à un simple épiphénomène social dans la mesure où ils attestent d'une claire perception de transition, même si elle est restée inachevée. Bouleversement ou révolution, Mai 68 a bien représenté une révolte charnière entre deux époques, marquée par une génération rebelle et engagée. Ce mouvement, qui voulait apporter une réponse à la désillusion de tous ceux qui en avaient assez de " travailler pour vivre et de ne vivre que pour travailler ", n'a pourtant pas réussi à les affranchir du poids du consumérisme moderne, d'une société du succès et du profit. Et, tant bien que mal, entre la gauche et la droite, aussi conservatrices l'une que l'autre, et l'utopie anarchiste de l'autogestion généralisée apparaissant tout aussi inappropriée, le défi démocratique finit par s'imposer, investissant dans un espace politique 'normalisé'. La société française est devenue politiquement et culturellement plus libérale qu'elle ne l'était avant. Avec ses conquêtes sociales ${ }^{8}$, juridiques et politiques ${ }^{9}$, elle est devenue aussi plus démocratique, plus égalitaire, aussi 
plus hédoniste (cf. Weber, 1998), comme l'ont bien remarqué aussi Alain Renaut ou Gilles Lipovetsky.

Mai 68 fut peut-être aussi l'expérience d'une déception, un rêve humaniste peut-être, mais qui a vu émerger, à sa place et comme par contrecoup, une société de consommation peu humaniste en fait ; c'est du moins ce que pensent Alain Renaut et Luc Ferry. Et Alain Finkielkraut de préciser que, dans cette sensation simultanée de rêve et d'échec, de réalisé et d'inachevé, il y a peut-être beaucoup de ce que Hannah Arendt appelait le « ressentiment » de l'homme moderne : ressentiment fondamental à " ne voir ni rime ni raison dans le monde tel qu'il se donne", et c'est pourquoi il «proclame ouvertement que tout est permis, et il croit secrètement que tout est possible» (Arendt, 1951: 438). Hannah Arendt pensait évidemment à ce "tout est possible » auné à la mesure des crimes commis au nom d'une humanité supérieure et qui a servi à les justifier. Alors que seule la gratitude est un sentiment capable de se présenter comme alternative au nihilisme de ce ressentiment: gratitude pour tout ce qui nous est donné et dont nous ne sommes pas les auteurs, à savoir la vie elle-même, le monde, la variété de la nature et du genre humain.

Une génération qui a hérité, comme mémoire récente, « deux guerres mondiales, les totalitarismes de droite et de gauche, hitlérisme et stalinisme, Hiroshima, le goulag, les génocides d'Auschwitz et du Cambodge (Levinas, 1991: 114), ne peut échapper au sentiment de révolte, à l'idée que plus rien n'a de sens, que l'homme même est absurde. Et pourtant, même si on a toutes les raisons pour continuer à se demander, l'air sinistre, s'il est encore possible d'écrire des poèmes après Auschwitz (cf. Adorno $)^{10}$, sans doute la pire des dérives humaines, mieux vaut cultiver la gratitude politique et reconnaitre qu'il est possible de se réconcilier avec la variété du genre humain, voire d'admirer les différences entre les hommes, car, comme le remarque encore Hanna Arendt, « ce sont des hommes et non l'homme qui habitent la terre » (Levinas, 1991: 439).

Certains pensent qu'il faudrait, peut-être, ajouter ce sentiment au répertoire des acquis de Mai 68 pour qu'il s'accomplisse vraiment. D'autres, toujours fidèles à l'utopie de mai 68 , considèrent plutôt qu'un « retour sur les désillusions qu'elle a occasionnées nous en restitue aujourd'hui la pertinence, dans son inachèvement même. Ça n'était 'qu'un début', en effet : il nous reste à inventer la suite » (Monteil, $1996: 26)$. Pour nous tous, enfin, Mai 68 est, en définitive, une page de l'histoire récente qui ne doit pas être tournée sans être lue attentivement. Mais il faut garder l'ouverture d'esprit, éviter la démagogie pédagogique et laisser aux générations, présentes et à venir, le soin de faire leur propre lecture et de définir leurs propres ambitions et désirs.

BIBLIOGRAPHIE

ARENDT, Hannah (1951). The Burden of Our Time, Londres, Secker and Warburg.

ARON, R. (1968). La Révolution introuvable, Paris, Fayard. 
CAMON, Ferdinando (1989). Conversations with Primo Levi, Marlboro, The Marlboro Press.

DEBRAY, Régis (1981). Critique de la raison politique, Paris, Gallimard.

FERRY, Luc, RENAUT, Alain (1988). La Pensée 68, Paris, Gallimard.

FINKIELKRAUT, Alain (2015). La Seule exactitude, Pairs, Stock.

FINKIELKRAUT, Alain (2013). L'Identité malheureuse, Paris, Stock.

FINKIELKRAUT, Alain (2005). Nous autres les modernes. Quatre leçons, Paris, Gallimard.

FINKIELKRAUT, Alain (1996). L'Humanité perdue. Essai sur le $\mathrm{xx}^{\mathrm{e}}$ siècle, Paris, Seuil.

FINKIELKRAUT, Alain (1987). La Défaite de la pensée, Paris, Gallimard.

KRISTEVA, Julia (1988). Étrangers à nous-mêmes, Paris, Gallimard, « folio essais ».

LÉVINAS, Emmanuel (1991), “La souffrance inutile”, in Entre Nous. Essais sur le Penser-à-l'autre, Paris, Grasset.

LIPOVETSKY, Gilles (1983), L'Ère du vide : essais sur l'individualisme contemporain, Paris, Gallimard.

LIPOVETSKY, Gilles (1988), « 'Changer la vie' ou l'irruption de l'individualisme transpolitique », in Pouvoirs, revue française d'études constitutionnelles et politiques, $n^{\circ} 39$ - Mai 68, p. 91-100. Consulté le 2018-04-06 15 : 38 : 33. URL: http://www.revue-pouvoirs.fr/Changer-la-vie-ou-l-irruption-de1.html

MONTEIL, Pierre-Olivier (1996). “Mai 68, une utopie obsolète ?”, in Autres Temps, Année 1996, pp. 26-34.

MORIN, Edgar (1988). « La commune étudiante » in Mai 68 : la brèche, Paris, Complexe.

TODOROV, Tzvetan (2017). Le Triomphe de l'artiste. La Révolution et les artistes ; Russie : 1917-1941,

Paris, Flammarion / Versilio.

WEBER, Henri (1998). Que Reste-t-il de Mai 68 ? Essai sur les interprétations des 'événements', Paris, Seuil.

\section{NOTES}

1. «Que reste-t-il de Mai 68 aujourd'hui? Ses effets, ont-ils encore une incidence sur notre monde actuel? » Ce débat entre Luc Ferry et Edgar Morin, animé par Michel Winock, s'est tenu sur le site François-Mitterrand de la BnF, le mardi 18 mars 2008. Avec les célébrations du cinquantenaire en 2018, il y a eu de nombreux autres débats.

2. Les Accords de Grenelle, conclus le 27 mai 1968 prévoient une augmentation de $35 \%$ du SMIG et une augmentation de $10 \%$ en moyenne des salaires réels. Ils prévoient aussi la création de la section syndicale d'entreprise, actée dans la loi du 27 décembre 1968.

3. Nicolas Sarkozy, Discours à Bercy (29/04/2007), publié et disponible sur https:// www.singulier.eu/

4. Cf. : http://www.gauchemip.org/spip.php?article3062 ; consulté le 24 mars 2018.

5. Il y a juste quelques mois, je regardais TV sport avec quelques jeunes passionnés de football, joueurs eux-mêmes; ils ont entre 14 et 16 ans. Or un de nos jeunes footballeurs interviewé après un match pendant lequel il a été l'auteur d'un but dit 'génial', et qui en plus a donné la victoire à son équipe, la jeune idole a raconté ainsi son haut-fait sportif (je traduis littéralement ses mots): « Notre entraîneur nous avait dit qu'il fallait gagner coûte que coûte ; puis je suis entré et dans un dribble bien pense j'ai pris le ballon, j'ai tiré de toutes mes forces, et j'ai marqué ce but. Et puis il fallait dominer jusqu'à la fin [...] et nous les avons enculés jusqu'à la dernière minute (en pt : 
fodemo-los até ao último minuto). ».

A mes côtés, les jeunes qui regardaient leur idole, ahuris, se disaient : « il est fou, ce mec ; [...] dire ça à la télé ? Ce n'est pas possible ! [...] Mais quel con ! Ça ne se dit pas à la télé ! ». La déclaration maladroite du jeune footballeur à la télé avait vraiment choqué, même ceux qui le répètent quotidiennement sans arrêt (mais sur le terrain et entre eux, pas à la télé !). Il parait que le jeune joueur n'est plus revenu à la télé commenter le moindre match, même quand il fait des miracles sur le terrain! Il paraît que la vulgarité choque encore et que la forme de l'expression compte au Portugal, heureusement.

6. Finkielkraut cite Debray (1981).

7. Finkielkraut, $2013: 23$ : «L'économie [...] n'occupe pas seule la place que la politique a laissée vacante, notre situation ne peut se résumer à l'effacement progressif du citoyen par le travailleur-consommateur, tout n'est pas business as usual : il y a aussi la discordance des usages. Aux experts qui croient accéder par des chiffres à la chair du réel et qui affirment - calculette à la main - que l'afflux des immigrés compense providentiellement la baisse de natalité du Vieux Continent, l'expérience répond que les individus ne sont pas interchangeables. Si identiquement soumis soient-ils à la logique de l'intérêt, ils ne sont pas coulés dans le même moule, ils n'ont pas la même manière d'habiter ni de comprendre le monde ».

8. Parmi les conquêtes sociales les plus marquantes qui ont modifié la condition ouvrière en France, on peut citer non seulement la mensualisation des salaires, mais aussi la reconnaissance de la section syndicale d'entreprise; il y a eu l'augmentation générale de $10 \%$ des salaires et de $35 \%$ du SMIG ; puis la création du Salaire Minimum Interprofessionnel de Croissance - SMIC - et les accords contractuels sur la formation permanente. Tous ces changements, et malgré la peur $\mathrm{du}$ patronat, loin de provoquer une catastrophe économique, ont provoqué l'augmentation du pouvoir d'achat; et cette 'injection' d'argent supplémentaire fut même à l'origine d'une croissance hors du commun.

9. Ces conquêtes, juridiques et politiques, ont libéralisé les rapports entre les sexes, mais aussi entre les générations comme entre les gouvernants et les gouvernés. On peut citer, parmi d'autres, la liberté de la contraception, l'instauration de l'autorité parentale conjointe sur les enfants, la possibilité pour les femmes d'ouvrir un compte en banque sans autorisation préalable du mari, le droit à l'égalité professionnelle entre hommes et femmes.

10. La première fois où Theodor $W$. Adorno aborde la question de la poésie après Auschwitz, c'est dans un article écrit en 1949 intitulé «Critique de la culture et société », repris ensuite dans le recueil Prismes (Prismen, 1955 ; 1986, pour la traduction française). Dans la conclusion de cet article, Adorno écrit: «La culture transparente pour le matérialisme n'est pas devenue plus sincère au sens du matérialisme, mais seulement plus vulgaire. Avec sa particularité, elle a perdu le sel de la vérité qui résidait jadis dans son opposition à d'autres particularités. Lorsqu'on lui demande les comptes qu'elle refuse de rendre, on fait le jeu d'une culture qui se donne des grands airs. Neutralisée et refaçonnée, toute la culture traditionnelle est aujourd'hui sans valeur : par un processus irrévocable, cet " héritage » hypocritement revendiqué par les Russes est dans une large mesure devenu inutile, superflu, camelote ; en la traitant comme telle, les profiteurs de la culture de masse peuvent s'en prévaloir en ricanant. Plus la société devient totalitaire, plus l'esprit y est réifié et plus paradoxale sa tentative de s'arracher à la réification de ses propres forces. Même la conscience la plus radicale du désastre risque de dégénérer en bavardage. La critique de la culture se voit confrontée au dernier degré de la dialectique entre culture et barbarie : écrire un poème après Auschwitz est barbare, et ce fait affecte même la connaissance qui explique pourquoi il est devenu impossible d'écrire aujourd'hui des poèmes. L'esprit critique n'est pas en mesure de tenir tête à la réification absolue, laquelle présupposait, comme l'un de ses éléments, le progrès de l'esprit qu'elle s'apprête aujourd'hui à faire disparaître, tant qu'il s'enferme dans une contemplation qui se suffit à elle-même » (Adorno, 1986 : 26). 


\title{
RÉSUMÉS
}

Faut-il achever mai 68 ? Depuis L'Humanité perdue (1996), en passant par Nous Autres les modernes (2005) et L'Identité malheureuse (2013), jusqu'à La Seule Exactitude (2015), Alain Finkielkraut ne cesse de faire des allusions au mouvement et à l'événement de mai 68. Dans ces écrits, il s'attache davantage à pointer leurs dérives qu'à exalter une pensée qui leur serait propre, et qu'il a par ailleurs du mal à reconnaitre. Pour autant, il n'en établit pas moins des correspondances entre certaines dérives soixante-huitardes, sociales et idéologiques, et ce qu'il estime être les souffrances de la France d'aujourd'hui. Partant de quelques-unes de ses critiques, nous essayerons, dans cette réflexion, de mieux les cerner et de problématiser certains détails de mai 68 auxquels il se réfère et dont la portée nous semble absolument actuelle.

Should we put May 1968 to rest? Ever since his (1996) L'Humanité perdue to his (2005) Nous Autres les modernes and even the more recent (2015) La Seule Exactitude, Alain Finkielkraut keeps revisiting the movement and the events of May 1968. He talks of its outcomes, more than a thought in itself which the author himself admits finds difficult to acknowledge. Despite that, the author draws some comparisons between the social and ideological outcomes of the May 1968 movement and what he considers to be constraints to present day France. Thus, starting off with some of his criticism, we have tried, with this reflection, to understand and consider some specific features of that momentous historical time, which are undeniably current.

\section{INDEX}

Mots-clés : Dérives, droits, liberté d'expression, mai 68, paradigmes

Keywords : outcomes, rights, freedom of speech, May 1968, paradigms

\author{
AUTEUR \\ JOÃO DOMINGUES \\ CLP - Université de Coimbra \\ jcosta[at]fl.uc.pt
}

